\title{
JOINT TOA/DOA WIRELESS POSITION LOCATION USING MATRIX PENCIL
}

\author{
Kambiz Bayat \\ Analog Devices Inc. \\ 777 Bay Street, 2303, Toronto, ON M5G 2C8, Canada \\ kambiz.bayat@analog.com
}

\author{
Raviraj S. Adve \\ Department of Elec. and Comp. Eng., University of Toronto, \\ 10 King's College Road, Toronto, ON, M5S 3G4, Canada \\ rsadve@comm.utoronto.ca
}

\begin{abstract}
In this paper, the Matrix Pencil algorithm is used to recover the time and angle of signal arrivals from a mobile device to a base station. Signal transmission within a basic BPSK system in a slowly fading frequency selective wireless environment is considered. A method similar to the matched-filter idea is applied to overcome the Matrix Pencil restriction in the number of signal directions that can be measured. The estimated times and angles of arrivals are used in a proposed hybrid time/direction of arrival position location scheme.
\end{abstract}

Keywords - Matrix Pencil, Position Location, TOA, DOA.

\section{INTRODUCTION}

Position location is a technique that determines the position of a mobile device, often in conjunction with some additional mapping or direction information, in a wireless communication system. There are numerous possible applications for location services in a wireless network system. Examples of such applications span from information services, such as weather notifications and traffic reports, to more important assistance services such as safety and security. In fact, locating 911 wireless phone callers (E911) has become mandatory in North America. Location-based services generally require high location accuracy in order to have acceptable performance in the market. As a result, the demand for high performance and cost effective position location technologies is increasing.

Detecting the time and angle of the signal arrival via the direct path between a mobile transmitter and base station receiver is the most important basis for the majority of technologies developed for location-based services. A precise time and direction of arrival measurement results in a more accurate location estimate. Regardless of the wireless system deployed, the performance of a location technology depends on accuracy, consistency, reliability, and the speed of the measurements of time and angle of signal arrivals.

In this paper, the Matrix Pencil algorithm [1,2] is proposed as a super-resolution parameter estimation technique for position location estimation. Matrix Pencil has some key advantages over the other super-resolution methods for parameter estimation such as MUltiple SIgnal Classification (MUSIC) [3,4]. Unlike other techniques, which generally need an estimation of the signal covariance matrix, Matrix Pencil deals directly with signal samples and does not require independent data samples. Furthermore, the Matrix Pencil algorithm requires less processing power and executes faster than some other super-resolution methods [3]. One of the advantages of this technique is that the correlation among the received signals does not have a significant impact on its performance and efficiency.

The superior performance of Matrix Pencil has been documented in previous works. In [4], Dharamdial et.al. compare Matrix Pencil to the MUSIC method in recovering the paths delays or TOAs (Time of Arrival) of a wireless multipath channel. And finally, the performance, complexity, as well as execution time of the Matrix Pencil technique for DOA (Direction of Arrival) estimation in CDMA cellular networks is compared to the MUSIC algorithm in reference [3].

The reminder of this paper is organised as follows. In the next section the Matrix Pencil algorithm for parameter estimation is developed. This technique is applied to the received signal samples for the purpose of TOA and DOA estimation. A procedure is also presented to extract the direct path signal from all the other unwanted received signals for DOA estimation. A joint TOA/DOA position location technique is presented in section III. Section IV presents simulation results to illustrate the performance of the algorithm. Some concluding remarks are provided in Section V.

\section{MATRIX PENCIL}

\section{A. Matrix Pencil Algorithm}

Consider the following exponential sequence of data of length $N$ described by

$y_{n}=\sum_{m=1}^{M} \alpha_{m} z_{m}^{(n-1)}+w_{n}, z_{m}=\exp \left(b_{m}+j x_{m}\right)$

in which $n=1,2 \ldots, N, \quad w_{n}$ represents noise, and $\alpha_{m}$ is the complex amplitudes of the $m^{\text {th }}$ signal of a total of $M$ signals. The desired signal parameters, which are to be estimated by the Matrix Pencil method, are $z_{m}, m=1, \ldots, M$.

The Matrix Pencil method begins by choosing a free parameter, $L$, known as the pencil parameter (or moving window length). This number is in the range 
$M \leq L \leq N-M$. The proper choice of the pencil parameter results in significant noise improvement. The Matrix Pencil method is most sensitive when this parameter is equal to $M$ or $N-M[1]$.

Now define matrix $Y$ from the sequence in Eqn. (1) as

$$
Y=\left[\begin{array}{ccccc}
y_{L+1} & y_{L} & \cdots & y_{2} & y_{1} \\
y_{L+2} & y_{L+1} & \cdots & y_{3} & y_{2} \\
\cdots & \cdots & \cdots & \cdots & \cdots \\
y_{N} & y_{N-1} & \cdots & y_{N-L+1} & y_{N-L}
\end{array}\right]_{(N-L) \times(L+1)}
$$

Then two matrices $Y_{1}$ and $Y_{2}$ are defined, in Matlab notations, as

$$
Y_{1}=Y(:, 1: L) \quad \text { and } Y_{2}=Y(:, 2: L+1)
$$

The matrix pencil for two matrices $Y_{1}$ and $Y_{2}$ is defined as a linear combination of the two matrices with scalar $\lambda$ described by $Y_{1}-\lambda Y_{2}$. In the absence of noise it is easy to verify that $Y_{1}$ and $Y_{2}$ can be decomposed into $Y_{1}=A Z B$ and $Y_{2}=A B$ such that:

$$
A=\left[\begin{array}{cccc}
1 & 1 & \ldots & 1 \\
z_{1} & z_{2} & \ldots & z_{M} \\
\ldots & \ldots & \ldots & \ldots \\
z_{1}^{N-L-1} & z_{2}^{N-L-1} & \ldots & z_{M}^{N-L-1}
\end{array}\right]_{(N-L) \times M} \times\left[\begin{array}{cccc}
\alpha_{1} & 0 & \ldots & 0 \\
0 & \alpha_{2} & \ldots & 0 \\
\ldots & \ldots & \ldots & \ldots \\
0 & 0 & \ldots & \alpha_{M}
\end{array}\right]_{(M \times M)}
$$

$B_{M \times L}=\left[\begin{array}{cccc}z_{1}^{L-1} & z_{1}^{L-2} & \ldots & 1 \\ z_{2}^{L-1} & z_{2}^{L-2} & \ldots & 1 \\ \cdots & \cdots & \ldots & \cdots \\ z_{M}^{L-1} & z_{M}^{L-2} & \ldots & 1\end{array}\right]_{(M \times L)}$

and

$$
Z_{M \times M}=\left[\begin{array}{cccc}
z_{1} & 0 & \ldots & 0 \\
0 & z_{2} & \ldots & 0 \\
\ldots & \ldots & \ldots & \ldots \\
0 & 0 & \ldots & z_{M}
\end{array}\right]_{(M \times M)}
$$

Provided that $M \leq L \leq N-M$, the pencil is of rank $M$. Under this condition each value of $\lambda=z_{m}$ is a rank reduing number of the pencil. However if $L$ is not within the above range, then none of $z_{m} \mathrm{~s}$ is a rank reducing number of the matrix pencil. This implies that the values of $z_{m}$ s are the generalized eigenvalues of the matrix pair $\left\{Y_{1}, Y_{2}\right\}$. It is proved in [1] that if the singular value decomposition of $Y_{2}$ is

$$
Y_{2}=U_{2} \Sigma_{2} V_{2}^{H}
$$

then the $M$ eigenvalues of the matrix defined as

$$
E_{2}=\sum_{2}^{-1} U_{2}^{H} Y_{1} V_{2}
$$

are exactly $z_{m}$ s (superscript ${ }^{H}$ denotes the Hermitian or conjugate transpose matrix operation).

The Total Least Squared Matrix Pencil approach [2] takes into account the effect of noise and measurement errors in eigenvalue calculation. We start with taking the singular value decomposition of $Y$ in Eqn. (2) as

$$
Y=U \sum V^{H}
$$

Assuming the singular values of $Y$ are sorted in descending order in $\Sigma$, then the matrix $Y^{\prime}$ is defined as

$$
Y^{\prime}=U(:, 1: M) \sum(1: M, 1: M) V(:, 1: M)^{H}=U^{\prime} \sum^{\prime} V^{\prime H}
$$

$Y$ is replaced by $Y^{\prime}$ in Eqn. (3) to derive $Y_{1}$ and $Y_{2}$, then the same eigenvalue calculation method is applied.

Note that the Matrix Pencil algorithm, described above, uses the data from a single snapshot directly. As explained later, if multiple data snapshots are available, an improved estimate can be formed. However, no attempt is made to estimate the second order statistics of the incoming signals.

\section{B. TOA Estimation Algorithm}

A wireless multipath channel containing $M$ path components can be modeled by:

$$
h(\tau)=\sum_{m=1}^{M} \alpha_{m} \delta\left(\tau-\tau_{m}\right)
$$

in which $\alpha_{m}$ and $\tau_{m}$ represent, respectively, the attenuation factor and propagation delay of the signal received on the $m^{\text {th }}$ path [6]. To estimate the path delays $\tau_{m}$ using the Matrix Pencil algorithm, the channel is studied in the frequency domain [4]. An optimum training sequence of length $P$ and symbol period $T$ is used for channel training [5]. The channel is considered to be slowly fading, meaning its components stay constant for the duration of the training sequence. The discrete frequency channel representation is 


$$
H_{p}=\sum_{m=1}^{M} \alpha_{m} z_{m}^{p}, \quad p=0,1,2, \ldots, P-1
$$

in which $H_{p}=H\left(p \omega_{0}\right), z_{m}=e^{-j \omega_{0} \tau_{m}}$, and $\omega_{0}=\frac{2 \pi}{P T}$.

This transfer function consists of the sum of $M$ discrete exponential frequency components. Therefore, the Matrix Pencil technique can be applied to estimate $z_{m} \mathrm{~s}$. Once the values of $z_{m}$ s are estimated, it follows that the estimates of delays are:

$$
\tau_{m}=-\frac{\mathfrak{J}\left\{\ln \left(z_{m}\right)\right\}}{2 \pi / P}
$$

where $\mathfrak{I}$ is the imaginary-part operator.

\section{DOA Estimation Algorithm}

Consider a uniform linear antenna array composed of $N$ identical omnidirectional sensors in a wireless channel with $M$ paths. The steering vector for each element can be expressed by

$a_{n}\left(\phi_{m}\right)=\exp \left(j \theta_{m}(n-1)\right), n=1,2 \ldots, N, m=1,2, \ldots, M$

in which $\theta_{m}=\frac{\omega_{0}}{c} d \sin \phi_{m}, \quad \phi_{m}$ is the angle of the $m^{\text {th }}$ incident signal, $d$ is the inter-element spacing, and $c=3 \times 10^{8} \mathrm{~m} / \mathrm{s}$ is the speed of light. At any sampling time, the received signal by the $n^{\text {th }}$ element can be represented by

$$
y_{n}=\sum_{m=1}^{M} \alpha_{m} z_{m}{ }^{(n-1)}+w_{n}, n=1,2 \ldots, N
$$

in which $z_{m}=\exp \left(j \theta_{m}\right)$ and $w_{n}$ is the Gaussian noise. Under the condition $M \leq N / 2$, the Matrix Pencil algorithm can be applied to estimate the values of $z_{m}$ s. It easily follows that the direction of arrival $\phi_{m}$ of each multipath component can be expressed by:

$$
\phi_{m}=\sin ^{-1}\left\{\frac{\mathfrak{I}\left(\ln \left(z_{m}\right)\right.}{d \omega_{0} / c}\right\}
$$

Clearly, the restriction $M \leq N / 2$ cannot be met in most practical situations as the number of array elements is limited and there is no control over the number of multipath components impinging the antenna array. To overcome this shortcoming, the following technique is presented. This method enhances the received signal that is carried over the particular path of interest among all the other received signals. The outcome of this technique is a signal with only one significant component, which represents the desired direction. Then the Matrix Pencil algorithm is applied to estimate the direction of this signal. In this technique the received signal at each antenna element at any sampling time is multiplied by conjugate of the signal of interest, which is known by the help of TOA information. Assuming that the signal of interest is carried over the $k^{\text {th }}$ path then $x_{n k}$, which is the newly defined received signal for the $n^{\text {th }}$ antenna element, can be represented by

$$
x_{n k}=\frac{1}{P} \sum_{p=1}^{P} b_{k}^{*}(p) y_{n}(p) \quad, n=1,2 \ldots, N, 1 \leq k \leq M
$$

Replacing $y_{n}(p)$ from Eqn. (15) further expands $x_{n k}$ as:

$$
x_{n k}=\frac{1}{P} \sum_{m=1}^{M} \sum_{p=1}^{P} b_{k}^{*}(p) A_{m}(p) b_{m}(p) a_{n}\left(\phi_{m}\right)+\frac{1}{P} \sum_{p=1}^{P} b_{k}^{*}(p) w_{n}(p)
$$

Extracting the $k^{\text {th }}$ component from the first term above results in:

$$
\begin{aligned}
x_{n k}=a_{n}\left(\phi_{k}\right) & \left\{\frac{1}{P} \sum_{p=1}^{P}\left|b_{k}(p)\right|^{2} A_{k}(p)\right\}+ \\
& +(\text { Multipath Interference })+(\text { Noise })
\end{aligned}
$$

Taking the statistical average of the terms in Eqn. (19) singles out the $k^{\text {th }}$ path in $x_{n k}$. A carefully selected set of modulated training symbols can reduce the effect of the (multipath interference) term in Eqn. (19).

\section{JOINT TOA/DOA POSITION LOCATION}

The hybrid TOA/DOA system presented here requires only one base station to locate a mobile device. At first, an optimum training sequence is transmitted from the mobile device to base station for the purpose of channel estimation. The frequency representation of the channel impulse response is then used by the Matrix Pencil algorithm to estimate the delays of each path components. In this case, in which the base station has an array of antennas, the time of arrivals are measured on each antenna element at the same time using the same training sequence. The final estimated time of arrivals are the average values of all the TOAs measured on each antenna sensor. The earliest arrival path is considered as the direct path signal. Note that this path does not necessarily generate the strongest response at the base 
station receiver. Then the enhancement technique explained in the previous section is applied to estimate the direction of arrival (DOA) of this direct path signal.

The TOA estimate defines a circle around the base station with radius $\mathrm{R}=\mathrm{TOA} \times c, c$ being the speed of light, and the DOA estimate defines a line of bearing that connects the base station and the mobile device. The intersection of the TOA circle and DOA line is the desired position location estimate. If the location of the mobile is at $\left(x_{M}, y_{M}\right)$ and the base station is located at $\left(x_{B S}, y_{B S}\right)$, then solving the following set of equations will result in an estimate for the mobile position location.

$$
\left[\begin{array}{l}
x_{M} \\
y_{M}
\end{array}\right]=\left[\begin{array}{l}
x_{B S} \\
y_{B S}
\end{array}\right]+\left[\begin{array}{c}
R \times \cos (D O A) \\
R \times \sin (D O A)
\end{array}\right]
$$

A geometric interpretation along with a linear least squares technique is used to solve this system (to simplify the calculations a 2D space is assumed).

In hybrid position location approaches, the strength of one technology compensates for the shortcomings of the other one. This fact makes the hybrid methods among the best techniques available for location estimation. The hybrid TOA/DOA method presented here is of particular interest because it eliminates the need for cooperating multiple distributed parties to solve the position location problem. The biggest advantage of this method is that it requires no more than one base station to provide an estimate for the position of a mobile. This technique could be an excellent candidate for indoor position location applications, since in a residential setting usually only one access point is installed. In the current CDMA wireless networks, due to power control, in some areas of the cellular region it is only the serving base station that can receive a clear signal from the mobile. As the other base stations receive a very weak signal from the mobile, they cannot take part in the position location process. This hybrid TOA/DOA method using one base station can address this issue as well.

\section{SIMULATION RESULTS}

Simulation programs are provided to demonstrate the performance of the hybrid position location method. The performance is evaluated by root mean square error (RMSE) of the position location estimates based on 10000 trials. For each run, a unique and random receiver noise and propagation multipath channel from the mobile to the base station is generated. After executing all trials, the mean and standard deviation of the location error are calculated as the measure of accuracy for position location.

The channel envelope is generated as a unit-power complex random Rayleigh process. The number of paths and the delay and angle of arrival associated with each path is set to known values. These are the channel characteristics to be estimated by the Matrix Pencil technique. The channel noise at the base station receiver antenna is modelled as a Gaussian random process with zero mean and variance of $\sigma^{2}=\frac{1}{2 S N R}$. The training sequence uses BPSK modulation.

The antenna array at the base station receiver consists of seven elements with equal inter-element spacing $d=\lambda / 2$. Choosing this value for the elements spacing results in maximizing the resolution of the DOA measurements.

As an example, a histogram of the position location estimates is illustrated in Fig. 1. The mobile is located at Cartesian distance $(4,6) \mathrm{km}$ from the base station and the base station is at origin. At $8 \mathrm{~dB}$ SNR, the average value for the location estimate is $(3.9821,6.0643) \mathrm{km}$ and the RMSE is $0.1587 \mathrm{~km}$. This location estimate results from the following TOA and DOA measurements. The direct path signal from the mobile device to the base station has the true delay of 3.0 seconds and true direction of -48.1873 degrees. The estimated TOA is 3.0683 seconds with RMSE 0.2362 seconds (normalized to period of the training sequence). The estimated DOA is -47.5693 degrees with 0.3851 degrees in RMSE.

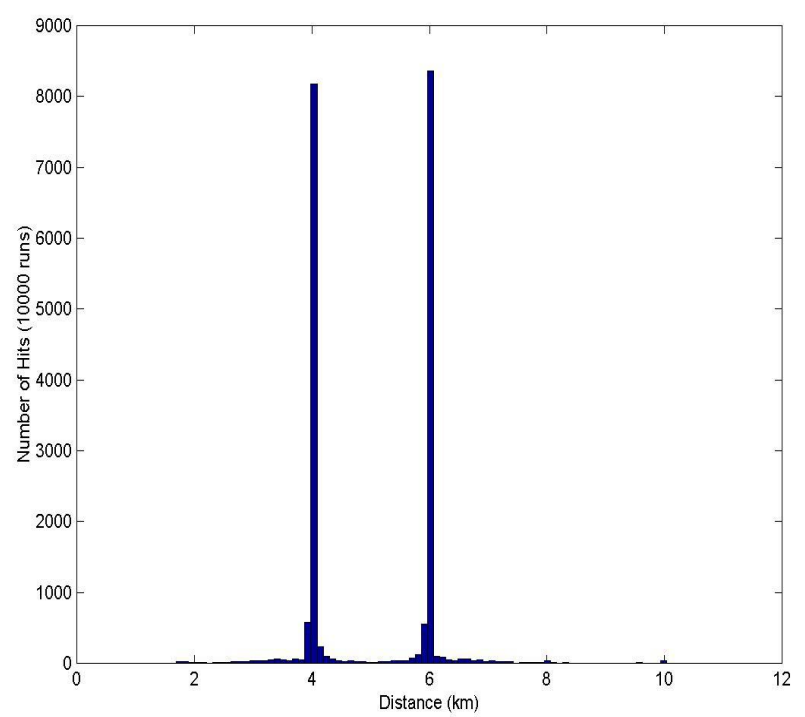

Fig. 1 Histogram of the location estimates.

To observe the effect of SNR in performance of the proposed scheme, the above procedure is repeated over a range of SNR values. Fig. 2 illustrates the amount of error in the location estimates at various SNRs.

The Matrix Pencil algorithm is a robust technique when the input signals are correlated. The same set of tests executed 
with introducing $30 \%$ correlation among the input signals. It was observed that the system was still able to estimate the location within a reasonable error. The performance results for this case are also reflected in Fig. 2.

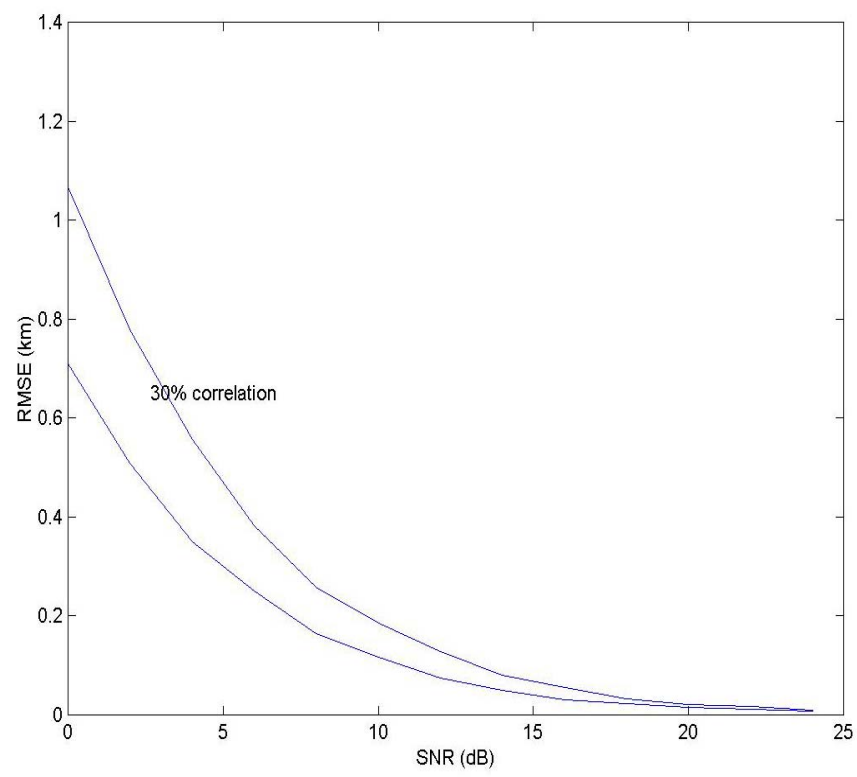

Fig. 2 RMSE in the location estimates in the joint TOA/DOA position location method.

\section{CONCLUSION}

The problem of position location estimation in a wireless network and the problem of time/direction of signal arrival measurement are closely linked. The Matrix Pencil algorithm for TOA and DOA estimation has several important advantages over other techniques such as MUSIC. The Matrix Pencil method is a super-resolution technique that takes advantage of the eigenstructure of incoming signals. Unlike the MUSIC method in which the eigenstructure is applied to the covariance matrix of received signals, Matrix Pencil deals directly with the input data. Since Matrix Pencil operates only on one snapshot of signals, it is computationally faster and less intensive than the MUSIC algorithm. The Matrix Pencil method performs efficiently when there is coherence among the received signals. The Total Least Squares Matrix Pencil technique is the practical way to use the Matrix Pencil algorithm in the presence of measurement errors and noise.

The proposed joint TOA/DOA position location technique, based on the Matrix Pencil method, can be used in wireless network systems in which no more than one base station or access point receiver is accessible or can be installed.

\section{REFERENCES}

[1] Y. Hua, and T.K. Sarkar, "Matrix Pencil Method for Estimating Parameters of Exponentially Damped /Undamped Sinusoids in Noise", IEEE Transactions on Acoustics, Speech, and Signal Processing, Vol. 38, No. 5, pp. 814-824, May 1990

[2] R.S. Adve, T.K. Sarkar, et al. , "Extrapolation of TimeDomain Response from Three-Dimensional Conducting Objects Utilizing the Matrix Pencil Technique", IEEE Transactions on Antennas and Propagation, Vol. 45, No. 1, January 1997

[3] C.K.E. Lau, R.S.Adve and T.K. Sarkar, "Mutual coupling compensation based on the minimum norm with applications in direction of arrival estimation", Accepted for publication, IEEE Trans. on Antennas and Propagation, to appear August 2004.

[4] N. Dharamdial, R.S. Adve, and R. Farha, "Multipath Delay Estimation using Matrix Pencil", Wireless Communications and Networking, Vol. 1, pp. 632-635, March 2003

[5] C. Tellambura, et al. , "Optimal Sequences for Channel Estimation Using Discrete Fourier Transform Techniques", IEEE Transactions on Communications, Vol. 47, No. 2, February 1999

[6] J. Proakis, Digital Communications, Fourth Edition, McGraw-Hill Inc., 2001

\section{ACKNOWLEDGEMENT}

Kambiz Bayat's graduate studies were actively supported by Analog Devices Inc., Toronto, ON, Canada. 